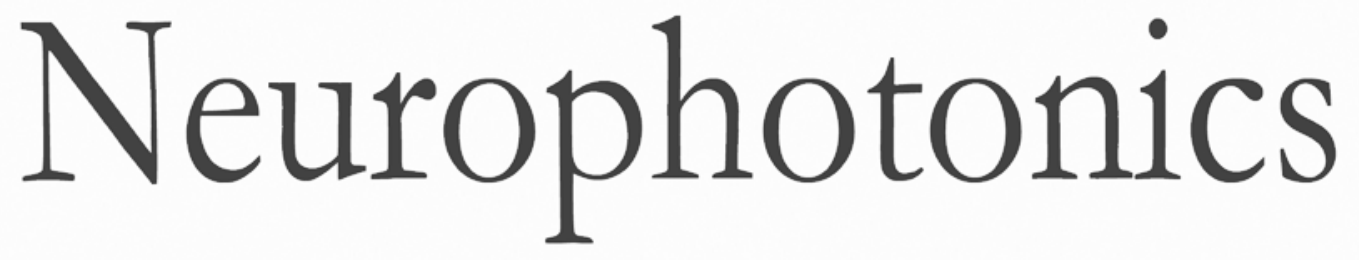

Voltage-sensitive dye imaging during functional development of the embryonic nervous system: a brief review with special thanks to Professor Larry Cohen

Yoko Momose-Sato

Katsushige Sato 


\title{
Voltage-sensitive dye imaging during functional development of the embryonic nervous system: a brief review with special thanks to Professor Larry Cohen
}

\author{
Yoko Momose-Sato a and Katsushige Satob,* \\ aKanto Gakuin University, College of Human and Environmental Studies, Department of Health and Nutrition, 1-50-1 Mutsuura-higashi, \\ Kanazawa-ku, Yokohama 236-8503, Japan

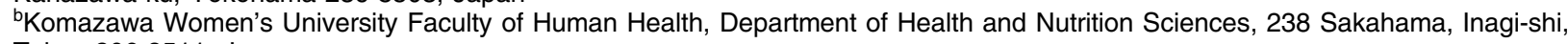 \\ Tokyo 206-8511, Japan
}

\begin{abstract}
Investigating the developmental organization of the embryonic nervous system is one of the major challenges in the field of neuroscience. Despite their significance, functional studies on the vertebrate embryonic central nervous system (CNS) have been hampered by the technical limitations associated with conventional electrophysiological methods. The advent of optical techniques using voltage-sensitive dyes, which were developed by Dr. Cohen and his colleagues, has enabled electrical activity in living cells to be monitored noninvasively and also facilitated the simultaneous recording of neural responses from multiple regions. Using optical recording techniques, it is now possible to follow the functional organization of the embryonic CNS and image the spatiotemporal dynamics involved in the formation of this neural network. We herein briefly reviewed optical studies on the embryonic CNS with a special emphasis on methodological considerations and the study of neuronal circuit formation, which demonstrates the utility of fast voltage-sensitive dye imaging as a powerful tool for elucidating the functional organization of the embryonic CNS. $\odot$ The Authors. Published by SPIE under a Creative Commons Attribution 3.0 Unported License. Distribution or reproduction of this work in whole or in part requires full attribution of the original publication, including its DOI. [DOI: 10.1117/1.NPh.2.2.021009]
\end{abstract}

Keywords: optical recording; voltage-sensitive dye; embryonic central nervous system; functiogenesis.

Paper 14078VSSR received Dec. 2, 2014; accepted for publication Dec. 31, 2014; published online Feb. 3, 2015.

\section{Introduction}

Investigating the developmental organization of the embryonic central nervous system (CNS) is one of the major challenges in the field of neuroscience. However, functional studies on the embryonic CNS have been hampered by the technical limitations associated with conventional electrophysiological methods. Since early embryonic neurons are small and fragile, the application of microelectrodes is often difficult. Furthermore, the simultaneous recording of electrical activity from multiple sites is limited, and, as a consequence, spatiotemporal patterns of neural network responses cannot be assessed.

Optical imaging with voltage-sensitive dyes (VSDs) has enabled the functional organization of the embryonic nervous system to be monitored and the spatiotemporal dynamics involved in the formation of this neural network to be imaged. This powerful technique was designed and developed by Dr. Cohen and his colleagues at Yale University and the Marine Biological Laboratory, and has been applied to various preparations from invertebrates to vertebrates (for reviews, see Refs. 1-6). The detection of transmembrane voltage changes from embryonic excitable cells, e.g., cardiac cells and neurons, with VSDs was initially achieved by Dr. Kamino and his colleagues in Tokyo Medical and Dental University (for reviews, see Refs. 7-9).

*Address all correspondence to: Katsushige Sato, E-mail: katsu-satoh@ komajo.ac.jp
We herein briefly reviewed the application of VSD recording to the embryonic CNS. We initially discussed several technical considerations and then presented some examples of VSD recording in the embryonic CNS.

\section{Technical Considerations}

The brain tissue of early embryos is small and thin. Furthermore, the embryonic brain is histologically loose with immature neurons and connective tissue. The immaturity of the embryonic brain has several advantages in optical recording. The high translucency of the preparation provides a large signal-to-noise ratio $(\mathrm{S} / \mathrm{N})$ in absorption measurements because the $\mathrm{S} / \mathrm{N}$ is proportional to the square root of the background light intensity if the dominant noise is shot noise. ${ }^{4,5}$ The immature cellularinterstitial structure allows the dye to diffuse readily from the surface to the deeper regions and, consequently, stains neurons relatively well. The simple structure and lower complexity of neural populations allows for the easier analysis of optical signal components.

However, the fragility and immaturity of embryonic neurons is disadvantageous for optical recording. Difficulties have been associated with directly comparing signals recorded optically with those measured electrophysiologically. Furthermore, when the meningeal tissue surrounding the brain is removed in order to stain the tissue with the dye, the roots of the cranial and spinal nerves are easily damaged. Postsynaptic potentials fatigue readily; therefore, signal averaging methods often cannot be employed. Since postsynaptic potentials also typically have 
a slow time course, the waveform of the optical signal is easily affected by various noise sources.

The high translucency of embryonic tissue provides a large $\mathrm{S} / \mathrm{N}$ in absorption measurements, and thus, absorption, rather than fluorescent, VSDs are commonly employed in embryonic preparations. In absorption measurements, signals arise from a baseline of high intensity transmitted light. Under this condition, a photodiode array is preferable to a charge coupled device or complementary metal oxide semiconductor image sensors. The optimal and most commonly used VSD in embryonic tissue is the merocyanine-rhodanine dye, NK2761. ${ }^{10}$ Although oxonol dyes, such as RH155 (NK3041) and RH482 (NK3630), give strong signals in other preparations, they are inferior to NK2761 for early embryonic preparations. NK2761 was designed by
Dr. Kamino as an analogue of Dye XXIII. ${ }^{11}$ Thus, advances in optical studies on embryonic preparations have benefited from the development of merocyanine dyes in early studies by Dr. Cohen and his colleagues.

\section{Application to the Study of Neuronal Circuit Formation}

We have applied VSD recording to the embryonic chick, rat, and mouse nervous systems, and examined the functional organization of neuronal circuits in the embryonic CNS (for reviews, see Refs. 9 and 12-15). The findings obtained revealed that postsynaptic responses in embryonic brainstem nuclei exhibited several common characteristics. (1) Synaptic transmission is mediated by glutamate, and $N$-methyl-D-aspartate (NMDA) and

Optical imaging of auditory responses in the chick embryo

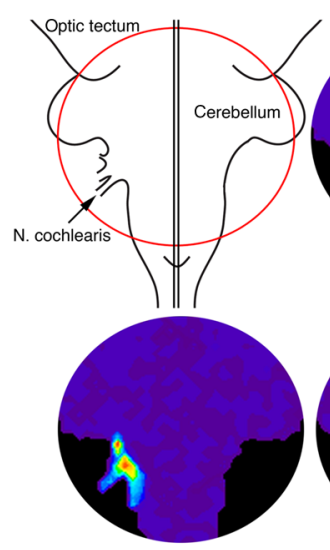

$20 \mathrm{~ms}$

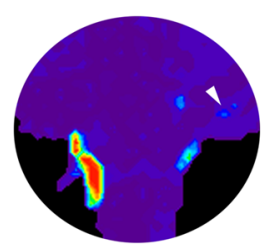

$60 \mathrm{~ms}$

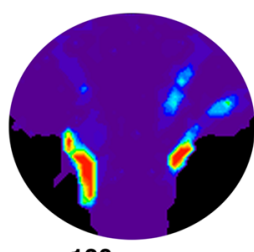

$130 \mathrm{~ms}$

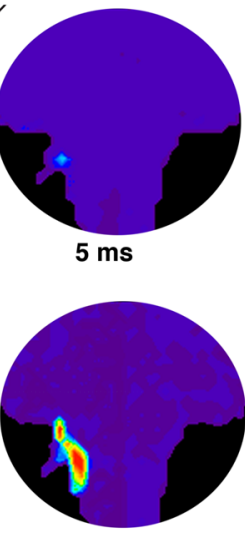

$45 \mathrm{~ms}$

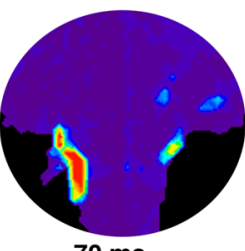

$70 \mathrm{~ms}$

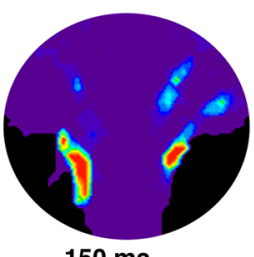

$150 \mathrm{~ms}$
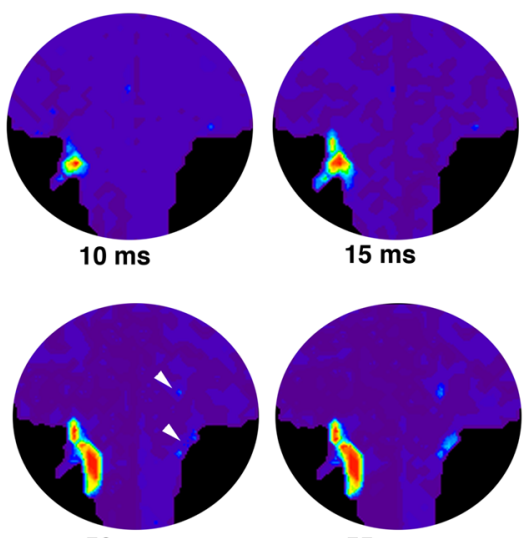

$50 \mathrm{~ms}$

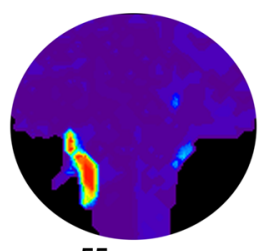

$55 \mathrm{~ms}$
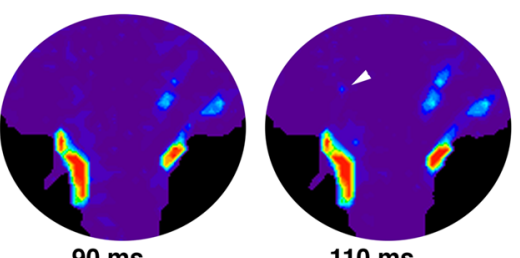

$110 \mathrm{~ms}$

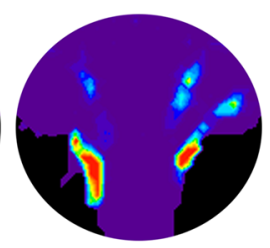

$170 \mathrm{~ms}$

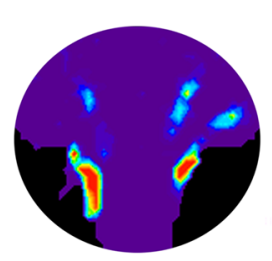

$190 \mathrm{~ms}$

5

$\left(\times 10^{-4}\right)$

Fig. 1 Time-lapse images of auditory responses in the chick embryo. Optical responses to stimulation of the auditory branch of the eighth cranial nerve (N. VIIIc) in an E8 chick brainstem are presented with pseudocolor images. The circular recorded region is shown in the top-left inset, and the time after $\mathrm{N}$. VIIlc stimulation is indicated below each image. Optical responses were first observed near the nerve entry zone in a region corresponding to the nucleus magnocellularis, nucleus angularis, and nucleus laminaris. Within $50 \mathrm{~ms}$, responses appeared in contralateral areas, corresponding to the contralateral nucleus laminaris and nucleus lemnisci lateralis (white arrowheads in the sixth frame). Within $60 \mathrm{~ms}$, responses were detected in the contralateral cerebellum (white arrowhead in the eighth frame). Finally, at $\sim 110$ ms after stimulation, responses were detected in the ipsilateral nucleus lemnisci lateralis (white arrowhead in the 11th frame). The projection to the contralateral cerebellum had been noted previously in anatomical studies and considered to be a transient anomaly that is later eliminated, but it had not been determined whether it ever became functional. As a demonstration of the power of the approach, the voltage-sensitive dye recording shows that the projection indeed generates a functional synaptic connection, although the function remains unclear. See Ref. 16 for more details. 
non-NMDA receptors are functionally expressed. (2) The initial phase of the excitatory postsynaptic potential (EPSP) is attributable to non-NMDA receptors, while the later phase is mediated by NMDA receptors. (3) The EPSP is very slow, with a duration on the order of seconds. (4) The EPSP readily fatigues; the amplitude of the EPSP decreases with repetitive stimuli of only $0.1 \mathrm{~Hz}$. (5) Synaptic function mediated by NMDA receptors is latently generated one day before the expression of EPSP, and the onset of synaptic function is regulated by an
$\mathrm{Mg}^{2+}$ block on NMDA receptors. (6) Functional synaptic connections are established at early stages before the morphological differentiation of pre- and postsynaptic neuronal populations has been completed.

Polysynaptic neuronal circuits have been optically identified in several systems, including the auditory, vestibular, vagal, olfactory, and spinal reflex pathways of the chick and the vagal system of the rat. The example of the chick auditory pathway shown in Fig. 1 indicates that cochlear nerve stimulation caused

\section{Spontaneous depolarization wave in the mouse embryo}

(a) Case 1

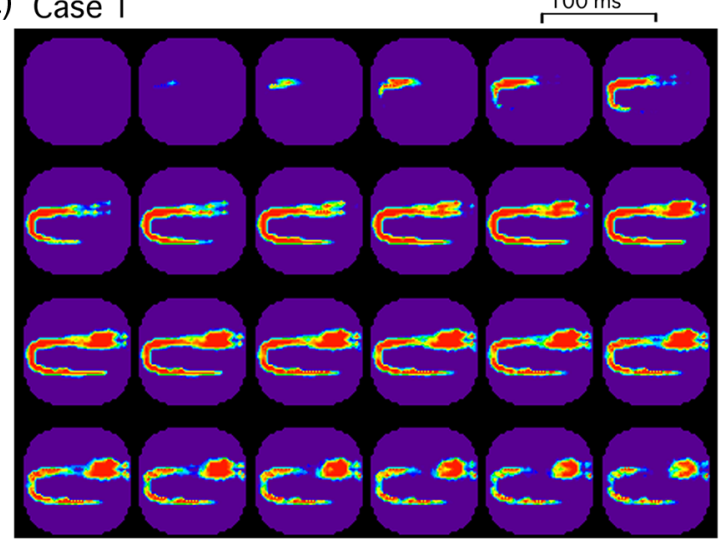

Case 2
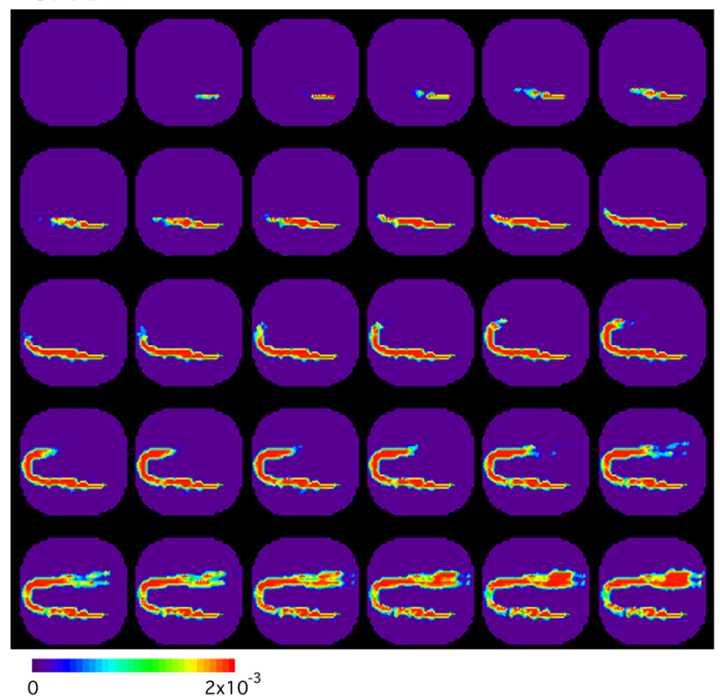

(b)

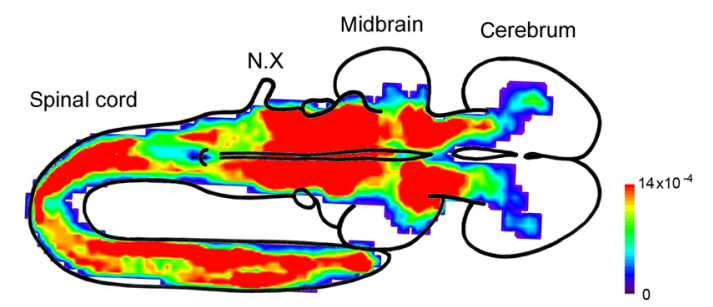

Fig. 2 Spontaneous depolarization wave in the mouse embryo. (a) Spatiotemporal patterns of the spontaneous depolarization wave in an E12 brainstem-spinal cord preparation. Images were acquired for case 1 and case 2 during two independent episodes in the same preparation. The signals on the right were detected from the five positions indicated in the lower-right inset. In case 1, activity was initiated in the upper cervical cord (green circle in the inset), while the wave originated in the lumbosacral cord in case 2 (red oval in the inset). (b) Color-coded representation of the maximum signal amplitudes of the spontaneous wave in an E13 mouse embryo. G.V, trigeminal ganglion; G.VIII, vestibulo-cochlear ganglion; N.X, vagus nerve. See Ref. 18 for more details. 
the sequential appearance of postsynaptic responses in the nucleus magnocellularis and nucleus angularis, and then in the nucleus laminaris and nucleus lemnisci lateralis. These findings demonstrated that (1) connections through higher-ordered nuclei have already been generated when or within one day after the afferents to the first-ordered nucleus are established (2) the basic adult connectivity pattern is apparent as soon as the connections become functional.

\section{Application to the Study of Correlated Wave Activity}

Cranial nerve stimulation often induces nonspecific, widely spreading wave-like activity during a specific period of development, suggesting that embryonic neurons are functionally correlated over a wide area (for reviews, see Refs. 14 and 17). The correlated wave was first observed with vagus nerve stimulation and was termed the depolarization wave. A significant feature of the wave is that a wide region of the CNS, including the spinal cord, medulla, pons, cerebellum, midbrain, and part of the cerebrum, is recruited across anatomical boundaries. This broadly extended propagation of the wave suggests that the depolarization wave may not serve as a simple regulator of the formation of specific neuronal circuits, but may play a more global role in the development of the CNS.

Consistent with this hypothesis, depolarization waves with similar spatial distributions were induced by stimulating various types of cranial and spinal nerves. Furthermore, the depolarization wave occurred spontaneously. These findings showed that the depolarization wave was triggered by multiple sources of external and endogenous activities, confirming that it was not specific to any neuronal circuit.

In addition to chicks, this depolarization wave has been observed in rat and mouse embryos, suggesting that the widely correlated wave is globally generated in different species, including mammals (Fig. 2). A comparison of depolarization waves between species revealed that they share several common characteristics. (1) In addition to being triggered by sensory inputs, the wave occurs spontaneously from the early stage of development, which is well before sensory pathways are

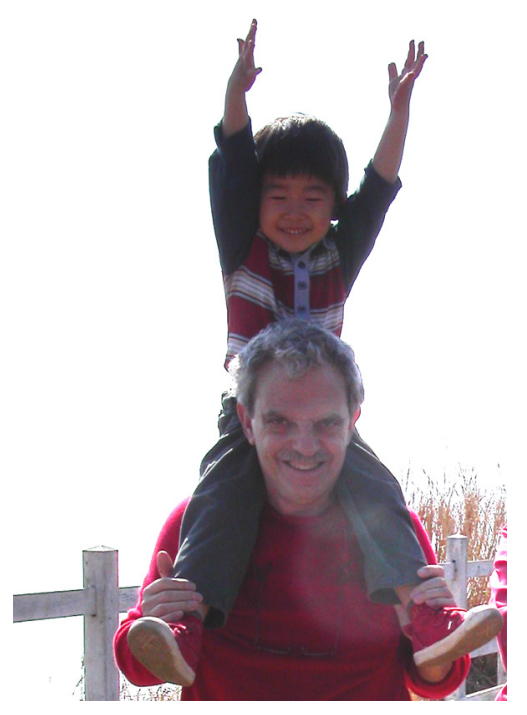

Fig. 3 Larry holding Muneaki Sato on his shoulders at the seashore in Chiba, Japan (2004). functionally organized. (2) The spontaneous wave is generated by multiple regions rather than a single, fixed pacemaker. (3) The wave is highly correlated across a large number of neurons and propagates over a wide region of the CNS. (4) The wave is mediated by multiple neurotransmitters and possibly gap junctions, and the primary mediator switches from acetylcholine to glutamate as development proceeds. (5) The wave is expressed during a particular period of development. (6) The wave is under homeostatic control, which may act to regulate and maintain the overall excitability of the network. The functional significance of the depolarization wave, especially the necessity of its large-scale coordination over a wide region of the CNS, has not yet been clarified, and deciphering this question is an important challenge for the future.

\section{Conclusion}

Optical recording techniques with VSDs have facilitated the characterization of neural responses in the embryonic nervous system. The ability to identify and image the spatiotemporal dynamics of developing neural circuits will allow the fundamental principles involved in the formation of this neural network as well as the functional organization of the embryonic nervous system to be elucidated in more detail.

\section{Acknowledgments}

We are grateful to Dr. Larry Cohen, Dr. Brian Salzberg, and Dr. Kohtaro Kamino for their warm and helpful instructions and support of our research. We also wish to acknowledge all of our collaborators at Tokyo Medical and Dental University, Kanto Gakuin University, and Komazawa Women's University. We particularly appreciate Dr. Brian Salzberg and Dr. Dejan Zecevic for editing this special issue. Since the initial application and introduction of voltage-sensitive dyes to embryonic tissue by Dr. Kohtaro Kamino, numerous studies have been devoted to elucidating the functional development and organization of the embryonic central nervous system (CNS). As discussed in the main text, optical analyses of the embryonic CNS have become possible by virtue of the pioneering and creditable achievements of Dr. Larry Cohen. Larry is not only an outstanding and honorable scientist, but also a warm and friendly person (Fig. 3). We would like to express our deepest respect and thanks to him.

\section{References}

1. L. B. Cohen, "Changes in neuron structure during action potential propagation and synaptic transmission," Physiol. Rev. 53, 373-418 (1973).

2. L. B. Cohen, "Historical overview and general methods of membrane potential imaging," Chapter 1 in Membrane Potential Imaging in the Nervous System: Methods and Applications, M. Canepari and D. Zecevic, Eds., pp. 1-11, Springer, NY (2010).

3. L. B. Cohen and B. M. Salzberg, "Optical measurement of membrane potential," Rev. Physiol. Biochem. Pharmacol. 83, 35-88 (1978).

4. B. M. Salzberg, "Optical recording of electrical activity in neurons using molecular probes," in Current Methods in Cellular Neurobiology, Vol. 3. Electrophysiological Techniques, J. L. Barker and J. F. McKelvy, Eds., pp. 139-187, Wiley, New York (1983).

5. A. Grinvald et al., "Optical imaging of neuronal activity," Physiol. Rev. 68, 1285-1366 (1988).

6. D. Zecevic and S. Antic, "Fast optical measurement of membrane potential changes at multiple sites on an individual nerve cell," Histochem. J. 30, 197-216 (1998). 
7. K. Kamino, "Optical approaches to ontogeny of electrical activity and related functional organization during early heart development," Physiol. Rev. 71, 53-91 (1991).

8. K. Kamino, A. Hirota, and H. Komuro, "Optical indications of electrical activity and excitation-contraction coupling in the early embryonic heart," Adv. Biophys. 25, 45-93 (1989).

9. Y. Momose-Sato, K. Sato, and K. Kamino, "Optical approaches to embryonic development of neural function in the brainstem," Prog. Neurobiol. 63, 151-197 (2001).

10. K. Kamino, A. Hirota, and S. Fujii, "Localization of pacemaking activity in early embryonic heart monitored using voltage-sensitive dye," Nature 290, 595-597 (1981).

11. L. B. Cohen et al., "Changes in axon fluorescence during activity: molecular probes of membrane potential," J. Memb. Biol. 19, 1-36 (1974).

12. Y. Momose-Sato and K. Sato, "Optical recording of vagal pathway formation in the embryonic brainstem," Auton. Neurosci. 126-127, 39-49 (2006).

13. Y. Momose-Sato and K. Sato, "The embryonic brain and development of vagal pathways," Resp. Physiol. Neurobiol. 178, 163-173 (2011).

14. Y. Momose-Sato, K. Sato, and K. Kamino, "Monitoring population membrane potential signals during functional development of neuronal circuits in vertebrate embryos," Chapter 8 in Membrane Potential Imaging in the Nervous System: Methods and Applications, M. Canepari and D. Zecevic, Eds., pp. 83-96, Springer, New York (2010).

15. J. C. Glover, K. Sato, and Y. Momose-Sato, "Using voltage-sensitive dye recording to image the functional development of neuronal circuits in vertebrate embryos," Dev. Neurobiol. 68, 804-816 (2008).
16. Y. Momose-Sato, J. C. Glover, and K. Sato, "Development of functional synaptic connections in the auditory system visualized with optical recording: afferent-evoked activity is present from early stages," J. Neurophysiol. 96, 1949-1962 (2006).

17. Y. Momose-Sato and K. Sato, "Large-scale synchronized activity in the embryonic brainstem and spinal cord," Front. Cell Neurosci. 7, 1-15 (2013).

18. Y. Momose-Sato, T. Nakamori, and K. Sato, "Spontaneous depolarization wave in the mouse embryo: origin and large-scale propagation over the CNS identified with voltage-sensitive dye imaging," Eur. J. Neurosci. 35, 1230-1241 (2012).

Yoko Momose-Sato is a professor and chairperson at Kanto Gakuin University. She received her MD and PhD in physiology from Tokyo Medical and Dental University. Her mentor was Dr. Kohtaro Kamino. She has applied the optical imaging technique with voltage-sensitive dyes to the embryonic nervous system and has investigated how the central nervous system is functionally generated during ontogenesis.

Katsushige Sato is a professor at Komazawa Women's University. He graduated from Tokyo Medical and Dental University Graduate School of Medicine. He was mentored by Dr. Kohtaro Kamino and received his $\mathrm{MD}$ and $\mathrm{PhD}$ in physiology. Using voltage-sensitive dye imaging, he has collaborated with Dr. Momose-Sato on projects of functiogenesis of the central nervous system. He also applied intrinsic optical imaging to the human brain during neurosurgical operations. 